\title{
The Research and Analysis of Parallax Error Decrease in Astronomical Navigation Positioning
}

\author{
Wu Jianhua, Zuo Mengjin, Zhao Xin, Ma Zhuang \\ Tianjin University of Technology, \\ Tianjin, P.R. China
}

\begin{abstract}
Improving the position precision will be the future development trend in astronomical navigation positioning, this article mainly introduces the influence of the parallax in the observation height of astronomical navigation positioning. Its producing principle and the corresponding error produced are analyzed and the correction to error is further researched. With the development of simulation technology, combining the simulation technology and astronomical navigation positioning accuracy analysis can make more accurate error analysis and then make navigation positioning precision and accuracy greatly improved.
\end{abstract}

Keywords-astronomical navigation, parallax eeror, simulation

\section{INTRODUCTION}

Astronomical navigation positioning is making use of marine equipment to observe the position of the stars and then to get the position of the ship by calculating the corresponding mathematical model. Therefore, celestial navigation has its unique advantages: The equipment is simple and reliable; Observation goal is natural objects and not from human's control; Don't launch any sound, light and radio and has concealment quality, etc. But because of some human's factors or external environment (such as sea conditions, eye high, temperature, pressure, visibility that is slow to change) or some other influential factors, the measurement height of object will produce certain error. Since parallax is one of the main factors that affect observation height and the resulting error may often be ignored. This article mainly analyzes the astronomical navigation positioning producing principle of parallax and the resulting error makes correction and corresponding simulation. Combining precision analysis and simulation technology makes astronomical navigation positioning accuracy improved, error further decreased and position more accurate.

\section{PARALLAX ANALYSIS}

The height of the object must be convered to the earth's globe height, so as to meet the astronomical navigation positioning principle of celestial height requirements. The difference of true celestial ground height $\angle \mathrm{BAH}$ and the geocentric height $\angle \mathrm{BOH}^{\circ}-\mathrm{p}$ calls parallax. It is shown in figure1.

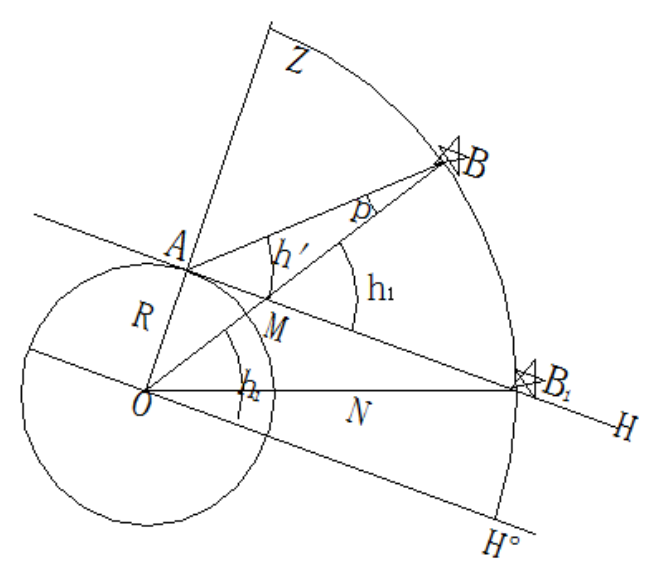

Figure1. Parallax principle

The main reason of celestial produced parallax is that the object is close to the earth and the light to the earth surface's observer and the geocentric light path is not parallel. Based on the above figure, we can get parallax correction as follows.

$\mathrm{AH} / / \mathrm{OH}^{\circ}, \mathrm{h} 1=\angle \mathrm{BOH}^{\circ}=\angle \mathrm{BMH}, \mathrm{h} 1$ is exterior angle. According to the sine theorem:

$$
\frac{R}{\sin \mathrm{p}}=\frac{N}{\sin \left(90+h^{\prime}\right)} \Rightarrow \sin p=\frac{R}{N} \cosh ^{\prime}
$$

Through the above formula: 
The closer the celestial body is, the bigger parallax is; on the contrary, parallax is smaller. For the stars, $\mathrm{N} \approx \propto, \mathrm{p}=0$.

The higher the object is, the smaller parallax is ; $\mathrm{h}^{\prime}=90$, parallax is zero.

The lower the object is, the bigger parallax is; $h^{\prime}=0$, parallax is called horizontal parallax $\mathrm{p}$.

In the spherical triangle $\mathrm{AB}_{1} \mathrm{O}$ :

$$
\sin \mathrm{p}^{\prime}=\frac{\mathrm{R}}{\mathrm{N}} \Rightarrow \sin \mathrm{p}=\sin \mathrm{p}^{\prime} \cosh { }^{\prime}
$$

Both $\mathrm{p}$ and $\mathrm{p}^{\prime}$ are small angle, we can make derivation:

$$
\mathrm{p}=\mathrm{p}^{\prime} \cosh \mathrm{h}^{\prime}
$$

Because the above parallax is mainly obtained in the average radius of the earth, the earth is the ellipsoid and the radius are different, we can make a measurement error in different sensible latitude.

\section{PARALLAX ERROR ANALYSIS AND SIMULATION}

In the earth's ellipsoid, a is semi-major axis, b is semiminor axis, e is eccentricity ratio, $\varphi$ is observed latitude. As is shown in figure2.

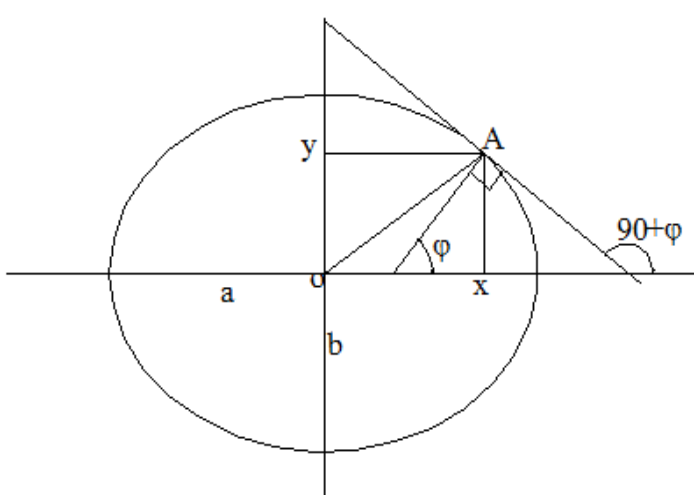

Figure2. Earth's ellipsoid

In the meridian equation of earth ellipsoid:

$$
\frac{x^{2}}{a^{2}}+\frac{y^{2}}{b^{2}}=1
$$

According to the formula:

$$
\begin{gathered}
\mathrm{x}=\frac{a \cos ^{2} \varphi}{\sqrt{1-e^{2} \sin ^{2} \varphi}} \\
y=\frac{a\left(1-e^{2}\right) \sin \varphi}{\sqrt{1-e^{2} \sin ^{2} \varphi}}
\end{gathered}
$$

Use the Pythagorean theorem:

$$
O A=\mathrm{R}_{\mathrm{T}}=\sqrt{x^{2}+y^{2}}=\frac{a \sqrt{\cos ^{4} \varphi+\left(1-e^{2}\right)^{2} \sin ^{2} \varphi}}{\sqrt{1-e^{2} \sin ^{2} \varphi}}
$$

Because the earth's average radius is calculated through the volume equallty. according to the formula:

$$
\frac{4}{3} \pi \mathrm{R}^{3}=\frac{4}{3} \pi \mathrm{a}^{2} \mathrm{~b} \Rightarrow \mathrm{R}=\sqrt[3]{\mathrm{a}^{2} \mathrm{~b}}
$$

The actual parallax correction should be:

$$
\operatorname{sinp}=\frac{\mathrm{R}_{\mathrm{T}}}{\mathrm{N}} \cosh ^{\prime}
$$

Using the formula(1) (2) (3) (8) (9), different observation latitude and different height make the error:

$$
\Delta \mathrm{p}=\left(\frac{R_{T}}{R}-1\right) p^{\prime} \cosh ^{\prime}
$$

In the shifting line positioning, the moon and the sun are used as stars. Maximum $\mathrm{p}^{\prime}$ appears horizon. We can use the formula(10), and use matlab to program, and then make use of matlab to simulat maximum error variation with different latitude varying. In the process of simulation, $h^{\prime}$ is zero, so we ensure that the Cosine take the maximum. The result of simulations of the sun and the moon is shown in the figure 3 and figure 4. 


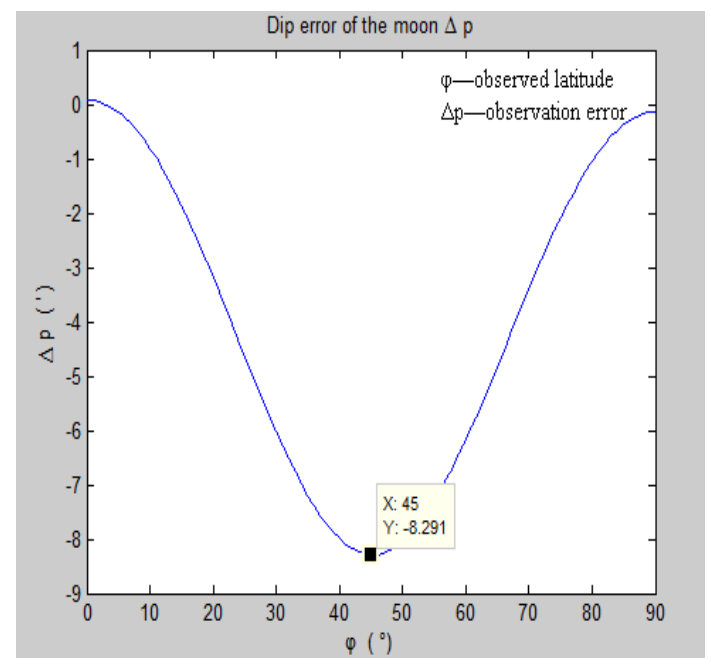

Figure3. The moon error variation with different latitude

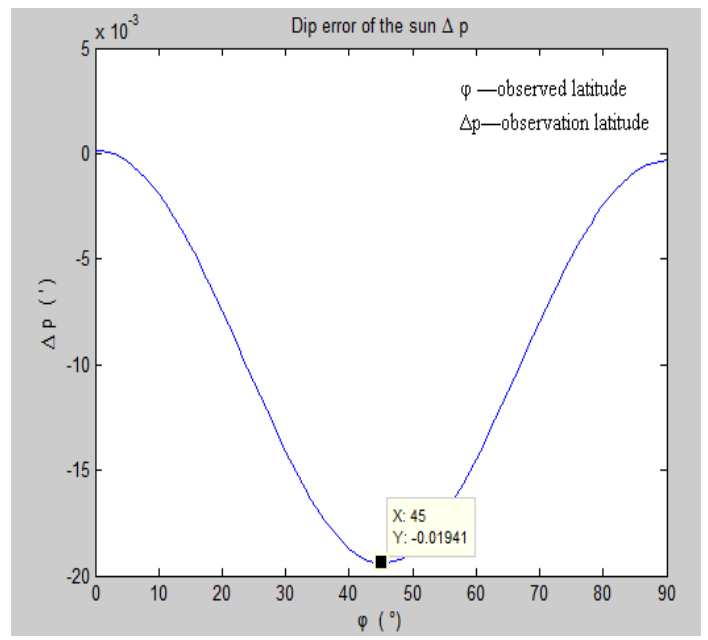

Figure4. The sun error variation with different latitude

According to the above figure, when latitude is between 0 and $45^{\circ}$, error will increase with the increasing of latitude; when latitude is between $45^{\circ}$ and $90^{\circ}$, error with the increasing of latitude will decrease; when latitude is equal to $45^{\circ}$, the maximum error appears. we can find that the sun's maximum error is about $0.02^{\prime}$ and the moon's maximum error is about $8.29^{\prime}$. In the process of localization, $1^{\prime}$ is equal to about $1 \mathrm{kn}$ and latitude effect on $1 \mathrm{kn}$ length is small. The moon that has greater positioning error is about $8 \mathrm{kn}$, so the process of calculation can make the positioning accuracy decrease and make the measured ship's position deviate from actual position. If the error is bigger, the sailing ship on the sea may be dangerous. Accurate positioning has important significance to celestial navigation. The simulation technology application will make it possible.
By using the formula (10), we could deduce formula that the biggest error is changing with different observation height in the same latitude as follow.

$$
\Delta \mathrm{p}=\mathrm{kp}^{\prime} \cosh { }^{\prime}
$$

As above formula, $\mathrm{k}$ is the obtained results of calculation in a certain latitude. For example $45^{\circ}$, we could simulat maximum error variation with observation height varying. The result of simulations of the sun and the moon is shown in the figure 5 and figure 6.

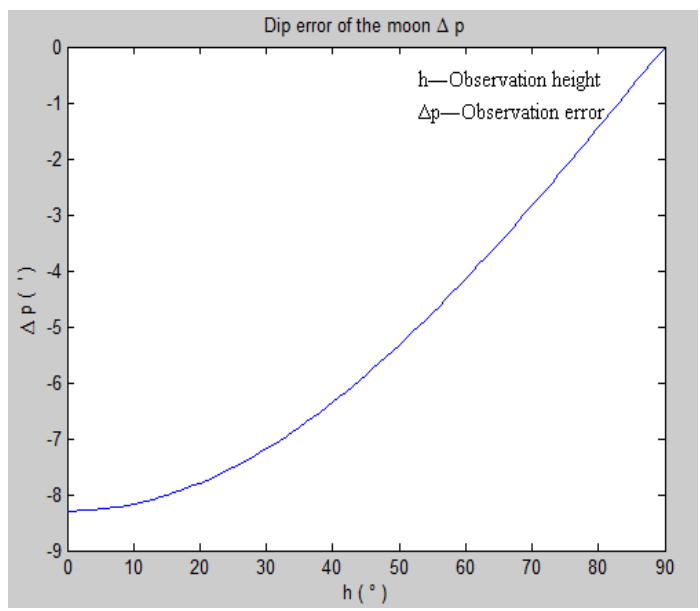

Figure5. The moon error variation with observation height

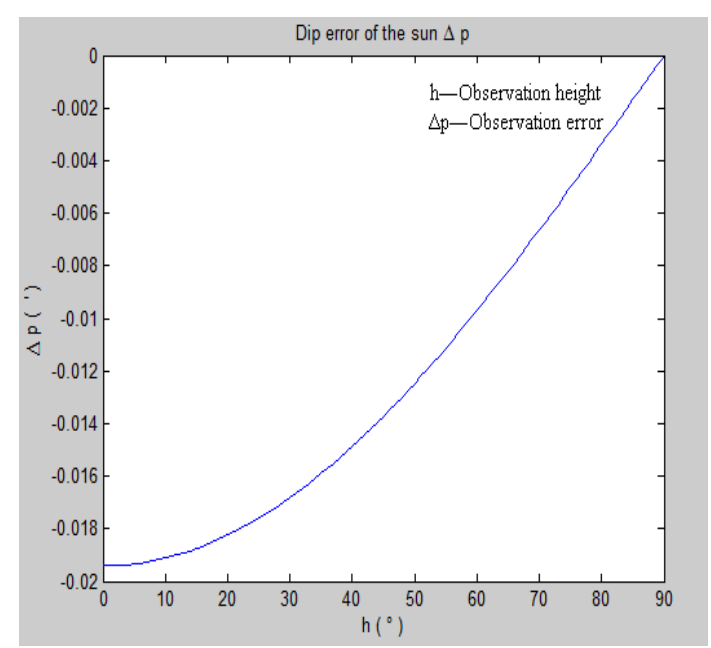

Figure6. The sun error variation with observation height

According to the above figures, when a latitude of observation is certain, the error with observation height 
increasing will decrease; when the observation height is zero, the error is biggest.

\section{EXAMPLE VERIFICATION}

Verification: On 2009.02.15, Tianren ship set off from the Tianjin Xingang Port to Inchon Port on the $\mathrm{ZT}_{1} 12^{\mathrm{h}} 30^{\mathrm{m}}$. The height of eye e $18 \mathrm{~m}$, index error and device difference $(\mathrm{i}+\mathrm{s})=0^{\prime} .0$. The course $\mathrm{CA} 99^{\circ}$, the speed V18kn, GMT1 $04^{\mathrm{h}} 30^{\mathrm{m}} 26^{\mathrm{s}}$, the first sun altitude $\mathrm{hs}_{1} 38^{\circ} 17^{\prime} .0$, the solar azimuth $\mathrm{A}_{1} 183^{\circ}$, GMT2 $06 \mathrm{~h} 17 \mathrm{~m} 40 \mathrm{~s}$, the second sun altitude $31^{\circ} 29^{\prime} .5$, solving the sun shift line position on ZT2 $14 \mathrm{~h} 18 \mathrm{~m}$ ?

we could check the nautical almanac to get the sun coordinates, Declination(Dec) and Greenwich hour angle(GHA):

h1 $38^{\circ} 24^{\prime} .6$ Dec1 $12^{\circ} 38^{\prime} .4$ S GHA1 $244^{\circ} 04^{\prime} .6$.

\section{h2 $31^{\circ} 36^{\prime} .8$ Dec2 $12^{\circ} 36^{\prime} .9$ S GHA2 $270^{\circ} 53^{\prime} .1$.}

Using spherical triangle calculate:

$\varphi 38^{\circ} 28^{\prime} .68 \mathrm{~N} \lambda 119^{\circ} 20^{\prime} .61 \mathrm{E}$

Using maximum error formula(10) through calculation:

$\Delta \mathrm{p} 1=0.0144{ }^{\prime}, \Delta \mathrm{p} 2=-0.0157^{\prime}$

Observation height after correction:

h1 $1^{\prime}=\mathrm{h} 1-\Delta \mathrm{p} 1=38^{\circ} 24^{\prime} .6144$

$\mathrm{h} 2^{\prime}=\mathrm{h} 2-\Delta \mathrm{p} 2=31^{\circ} 36^{\prime} .5957$

Using spherical triangle calculate:

$\varphi 38^{\circ} 28^{\prime} .67 \mathrm{~N} \lambda 119^{\circ} 20^{\prime} .608 \mathrm{E}$

GPS data:

$\varphi=38^{\circ} 24^{\prime} .7 \mathrm{~N}, \lambda=119^{\circ} 15^{\prime} .9 \mathrm{E}$

Through the comparison, the corrected result gets closer to the actual ship position than the no correction, Error is about 0.01 ', $0.01 \mathrm{kn}$. Positioning accuracy is more accurate and the sailing on the sea is more safe.

\section{V.CONCLUSION}

Matlab has good numerical ability and excellent data visualization ability. The paper will make error analysis and matlab simulation technology combined, we can clearly analyse the changing rule of the error and size changing. Through the correction, the error influence on the navigation positioning will gradually decrease and it improve the navigation accuracy and promote the development of astronomical navigation positioning. We could conduct simulations of the other errors of astronomical navigation that makes a more accurate precision analysis. Based on the calculation of error, we can get a more accurate observation height and then get a more accurate ship position. At last, high-accuracy precision navigation becomes possible and benefits the progress of celestial navigation.

\section{REFERENCES}

[1] Zhang Zhichong. Proficient in matlab. The Beijing university of aeronautics \&astronautics press, 2011.11.01

[2] Navigation Study / Editor-in-Chief Dingyong. Dalian Maritime University Press, 2000.9

[3] Yu Kaifeng, Wu Dewei, Qi Junyi, Celestial navigation technology for military application and development, national defense technology foundation, 2006

[4] Zhu Xiaohong, $\mathrm{Xu}$ rui, Zhang Junyan, The current construction situation and future development of Celestial navigation standard system, Geospatial information, 2011.6

[5] Ma zhuang, Tianjin University of Technology. Calculate astronomical positions using spherical trigonometry. 7th Navigation Technology, 2004

[6] Purple Mountain Observatory, Chinese Academy of Science. The Chinese Astronomical Calendar for 2009Year [M ]. Beijing Publishing House of Science, 2009.(in Chinese) 